\title{
Is fertility sparing surgery a treatment option for premenopausal patients with dysgerminoma?
}

\author{
Iavazzo $\mathrm{C}^{1}$, Vorgias $\mathrm{G}^{2}$, Iavazzo $\mathrm{PE}^{3}$, Gkegkes $\mathrm{ID}^{4}$ \\ Gynaecological Oncology Department, Northampton General Hospital, Northampton, United Kingdom. \\ christosiavazzo@hotmail.com
}

\begin{abstract}
Dysgerminoma is the most common ovarian germ cell type ovarian tumour. Primarily, it presents in young women at reproductive age and thus, the preservation of fertility is considered to be fundamental when it is possible for these patients. In comparison to the past the restriction of the extent of the surgical procedure as well as the introduction of innovative chemotherapeutic regimens improved significantly both, the prognosis and the clinical outcomes of this rare neoplasia. As dysgerminomas are extremely radio- and chemosensitive, fertility sparing approach and less aggressive operations should be favoured. We present a narrative review of the multispecialty fertility sparing surgical and medical approach for women with dysgerminoma (Ref. 21). Text in PDF www.elis.sk. KEY WORDS: dysgerminoma, ovarian germ cell tumor, fertility sparing surgery, premenopausal, treatment.
\end{abstract}

\section{Introduction}

From all gyneacological neoplasms ovarian dysgerminoma represents a relative rare entity $(<2 \%)(1,2)$. Dysgerminoma predominantly affects adolescents and young women being less than 30 years of age at the time of diagnosis (3). In the majority of cases with ovarian dysgerminoma, the affected women will present with clinical stage IA (4). The presence of lymph node metastasis represents an independent predictor of poor survival (5). Ovarian dysgerminoma is extremely radio- and chemosensitive, thus the prognosis is generally excellent even in cases with advanced disease, with an overall survival at 5-year period of over $90 \%(3,6)$.

The young age of the patients, the rarity of the ovarian dysgerminoma and the absence of randomized trials are factors that influence the final treatment options. The aim of this narrative review is to give some answers on the utilization of fertility sparing surgery as a treatment option for premenopausal patients with dysgerminoma.

\section{Methods}

We included studies selected after performing a broad electronic search in the PubMed (27/07/2016) and Scopus (27/07/2016). The search strategy adopted in this narrative review included the combination of the following keywords: treatment AND fertility

${ }^{1}$ Gynaecological Oncology Department, Northampton General Hospital, Northampton, United Kingdom, ${ }^{2}$ Gynaecological Oncology Department, Metaxa Cancer Hospital, Piraeus, Greece, ${ }^{3}$ Department of Paediatrics, Rethymno Hospital, Rethymno, Crete, Greece, and ${ }^{4}$ First Department of Surgery, General Hospital of Attica "KAT", Athens, Greece

Address for correspondence: $\mathrm{Ch}$. Iavazzo, MD, MSc, PhD, 38, Seizani Str., Nea Ionia, 14231 Athens, Greece.

Phone: +306948054119
AND dysgerminoma. The references of the included studies were also hand-searched for additional studies. Studies written in languages other than English were not included. In order to limit the search range, only studies written after 1990 were considered for this review. Our search retrieved 9 studies which were included as eligible for our review. Studies reporting data on fertility sparing management in premenopausal patients with dysgerminoma were regarded as eligible for this review. Abstracts, conference papers, commentaries, animal studies, review articles as well as editorials were excluded from this review.

\section{Discussion}

Ovarian dysgerminomas occur in pure or mixed form with other types of germ cell tumours such as embryonal cancer, yolk sac tumour, choriocarcinoma and teratoma. Dysgerminomas are also associated with gonadoblastomas in about $50 \%$ of cases. It should be mentioned that $30 \%$ of the cases can occur in patients with dysgenetic gonads. Dysgerminomas are usually unilateral tumours $(90 \%)$ and $70 \%$ of the cases are diagnosed early with stage I disease. However, it should be noted that lymphatic spread to the pelvic and para-aortic lymph nodes can occur very early (7). Recurrences occur within 2 years of diagnosis and are curable (8).

Ovarian dysgerminoma presents histological similarities to testicular seminoma and for that reason the existing chemotherapeutic substances were firstly applied and also were effective in patients with seminoma $(9,10)$. In particular, dysgerminoma in comparison with other types of germ cell tumours, because at initial diagnosis it is more probable to be at stage Ia, is both chemo- and radiosensitive, the bilateral involvement is more common, from 10 to $15 \%$, while retroperitoneal lymph nodes are more frequently involved than the intraperitoneal ones, in case of lymphatic dissemination (11). 
The development of cisplatin-based chemotherapeutics has restricted the extent of the surgical excision offering the patient the possibility of fertility preservation. Fertility-sparing surgery with unilateral salpingo-oophorectomy has become the standard procedure in cases of dysgerminoma. Resection or biopsy is indicated in areas suspected to be involved by the tumour. In particular, biopsy of contralateral ovary might be considered in patients with dysgerminoma (even though there is a probability of secondary infertility related to post-operative adhesions) due to either occult or microscopic tumour dissemination (10-15\%) (12).

Almost $75 \%$ of the patients with dysgerminoma will present with clinical stage Ia disease (3). In the past, the majority of these patients were treated post-operatively with radiotherapy. Nevertheless, radiotherapy at pelvis and paraaortic nodes is correlated with a high incidence of ovarian dysfunction or/and sterility, and thus radiotherapy has been recommended at more advanced stage of the disease (13). In addition, the extent of radiation field is in direct correlation with the involved area. Also, the concept of prophylactic mediastinal radiotherapy does not seem to offer any advantage on evolution of the disease and it causes additional toxicity and increases the costs of treatment (11).

The above mentioned facts can lead to fertility sparing type of surgery with excellent oncological and good reproductive outcomes. More specifically, these patients after informed consent can undergo unilateral salpingo-oophorectomy with omental and peritoneal biopsies plus or minus biopsy of the contralateral ovary and pelvic and or para-aortic sampling/dissection in cases with lymph node involvement $(14,15)$. In cases of bilateral ovarian involvement, uterine preservation should be considered and IVF protocols with donor eggs could be used. According to Sigismondi et al, in dysgerminoma histology, residual disease could be left to spare fertility (16). Stage Ia tumours do not need adjuvant treatment. However, chemotherapy with schemes such as PEB (cisplatin, etoposide, bleomycin) or JEB (carboplatin, etoposide, bleomycin) or PVB (cisplatin, vinblastine, bleomycin) should be offered in patients with higher stage disease $(12,15)$. Several pregnancies have been reported after surgery and chemotherapy. It was shown that pregnancy outcomes and menstrual function after fertility sparing surgery for pure ovarian dysgerminomas are sufficient (17). In a recent study, 16 out of 50 patients who received fertility sparing surgery (32\%) achieved pregnancy with 14 live births (1). Brewer et al showed that after chemotherapy, $71 \%$ of patients maintained their normal menstrual function during and after chemotherapy, and $93 \%$ had returned to their pre-chemotherapy menstrual pattern while $35 \%$ achieved pregnancy (18). Radiotherapy of the pelvic and para-aortic lymph node areas is rarely used as first line treatment (15). Ovarian transposition of the contralateral ovary is proposed in such cases.

Patients with stage Ia tumours have $95 \%$ five-year disease free survival. The most common areas of recurrence in other stages are the peritoneal cavity and retroperitoneal lymph nodes which highlights the role of pelvic and/or para-aortic lymph node dissection in the fertility sparing approach of such patients. Patients with incomplete staging could undergo surgical restaging or surveillance. More specifically, a recent study showed that only $19.2 \%$ of patients with fertility sparing management had complete surgical staging which included lymph node dissection in $38.5 \%$, peritoneal and/or omental biopsies in $46.2 \%$ and peritoneal washings in $65.4 \%$ (19). The overall recurrence rate in this group of patients was $11.5 \%$ and it should be noted that all recurrences (mainly in peritoneal cavity or retroperitoneal nodes) occurred in patients who had incomplete staging. All patients with recurrence were cured by salvage therapy (19).

Advanced stage of the tumour $(\mathrm{p}=0.001)$ (which is correlated with treatment failure), histologic type $(\mathrm{p}=0.0004)$, residual tumour after salvage surgery $(p=0.0014)$ as well as high-dose chemotherapy after primary chemotherapy failed $(p=0.0405)$ were negative prognostic factors for the overall survival (20). Furthermore, the elevation of serum markers b-HCG ( $\beta$-human chorionic gonadotrophin $)$ and AFP $(\alpha$-fetoprotein $)(p=0.009)$ and the nonplatinum chemotherapy $(\mathrm{p}=0.003)$ were significantly associated with relapse whereas the age of the patient at the time of diagnosis has no prognostic value $(12,21)$.

From the above reviewed results, we suggest that the gold standard treatment of patients with dysgerminomas should be fertility sparing. Management of such patients should be performed in tertiary centres by subspecialists in gynaecological oncology after discussion in MDTs (multidisciplinary team - meetings) and obtaining informed consent from the patients. Genetic counselling is also proposed for such patients.

\section{Conclusion}

In conclusion, fertility sparing treatment should be considered as the standard care in case of young patients with ovarian dysgerminoma. In this therapeutic direction assisted the biological characteristics of the tumour, the evolution of the surgical techniques and principally the development of innovative chemotherapeutics. Nevertheless, a multidisciplinary approach that includes a variety of specialists such as gynaecologic oncologists, medical oncologists, psychooncologists, assisted reproduction specialists and paediatricians is essential. Larger, multicenter clinical trials may provide additional clarifications on the management and the treatment options.

\section{References}

1. Husaini ALH, Soudy H, El Din Darwish A et al. Pure dysgerminoma of the ovary: a single institutional experience of 65 patients. Med Oncol 2012; 29: 2944-2948.

2. Williams SD, Gershenson DM, Horowitz CT. Ovarian germ cell tumors. In: Hoskins WJ, PerezCA, Young RC (Eds). Principles and practice of gynecologic oncology. Philadelphia: Lippincott-Raven Publishers, 1997, 987-1001.

3. Quirk JT, Natarajan N. Ovarian cancer incidence in the United States, 1992-1999. Gynecol Oncol 2005; 97: 519-523.

4. De Palo G, Pilotti S, Kenda R et al. Natural history of dysgerminoma. Am J Obstet Gynecol 1982; 143: 799-807.

5. Kumar S, Shah JP, Bryant CS et al. The prevalence and prognostic impact of lymph node metastasis in malignant germ cell tumors of the ovary. Gynecol Oncol 2008; 110: 125-132. 


\section{$738-740$}

6. Smith HO, Berwick M, Verschraegen CF et al. Incidence and survival rates for female malignant germ cell tumors. Obstet Gynecol 2006; 107: 1075-1085.

7. Iavazzo C, Kondi-Pafiti A. Germ Cell Tumors, In: Non Epithelial Ovarian tumors. Clinicopathological issues, Nova Science Publishers 2013, 32-39.

8. Vicus D, Beiner ME, Klachook S, Le LW, Laframboise S, Mackay H. Pure dysgerminoma of the ovary 35 years on: a single institutional experience. Gynecol Oncol 2010; 117: 23-26.

9. Gershenson DM, Wharton JT, Kline RC, Larson DM, Kavanagh JJ, Rutledge FN. Chemotherapeutic complete remission in patients with metastatic ovarian dysgerminoma. Potential for cure and preservation of reproductive capacity. Cancer 1986; 58: 2594-2599.

10. Stanton GF, Bosl GJ, Whitmore WF, Jr, et al. VAB-6 as initial treatment of patients with advanced seminoma. J Clin Oncol 1985; 3: 336-339.

11. Pectasides D, Pectasides E, Kassanos D. Germ cell tumors of the ovary. Cancer Treat Rev 2008; 34: 427-441.

12. Gershenson DM. Management of early ovarian cancer: germ cell and sex cord-stromal tumors. Gynecol Oncol 1994; 55: S62-72.

13. Mitchell MF, Gershenson DM, Soeters RP, Eifel PJ, Delclos L, Wharton JT. The long-term effects of radiation therapy on patients with ovarian dysgerminoma. Cancer 1991; 67: 1084-1090.
14. Casey AC, Bhodauria S, Shapter A, Nieberg R, Berek JS, FariasEisner R. Dysgerminoma: the role of conservative surgery. Gynecol Oncol 1996; 63: 352-357.

15. Tangir J, Zelterman D, Ma W, Schwartz PE. Reproductive function after conservative surgery and chemotherapy for malignant germ cell tumors of the ovary. Obstet Gynecol 2003; 101: 251-257.

16. Sigismondi C, Scollo P, Ferrandina G et al. Management of bilateral malignant ovarian germ cell tumors: a MITO-9 retrospective study. Int J Gynecol Cancer 2015; 25: 203-207.

17. Boran N, Tulunay G, Caliskan E, Kose MF, Haberal A. Pregnancy outcomes and menstrual function after fertility sparing surgery for pure ovarian dysgerminomas. Arch Gynecol Obstet 2005; 271: 104-108.

18. Brewer M, Gershenson DM, Herzog CE, Mitchell MF, Silva EG, Wharton JT. Outcome and reproductive function after chemotherapy for ovarian dysgerminoma. J Clin Oncol 1999; 17: 2670-2675.

19. Mangili G, Sigismondi C, Lorusso D et al. Is surgical restaging indicated in apparent stage IA pure ovarian dysgerminoma? The MITO group retrospective experience. Gynecol Oncol 2011; 121: 280-284.

20. Lai CH, Chang TC, Hsueh S et al. Outcome and prognostic factors in ovarian germ cell malignancies. Gynecol Oncol 2005; 96: 784-791.

21. Murugaesu N, Schmid P, Dancey G et al. Malignant ovarian germ cell tumors: identification of novel prognostic markers and long-term outcome after multimodality treatment. J Clin Oncol 2006; 24: 4862-4866.

Received August 8, 2016. Accepted August 22, 2016. 